\title{
PROBABILISTIC ESTIMATION OF THE TIME-TO-REHABILITATION FOR DETERIORATING BRIDGES
}

\author{
Filippos Alogdianakis, Dimos C. Charmpis and Ioannis Balafas \\ Department of Civil and Environmental Engineering, University of Cyprus, \\ 75 Kallipoleos Str., P.O. Box 20537, 1678 Nicosia, Cyprus, \\ E-mails: alogdianakis.filippos@ucy.ac.cy, charmpis@ucy.ac.cy, ibalafas@ucy.ac.cy
}

Keywords: Rehabilitation, maintenance, bridge stock, aging, deterioration, corrosion, deicing salts, humidity, Weibull distribution.

\begin{abstract}
Being able to reliably predict the time-to-rehabilitation of aging bridges is a major issue in cost-effectively maintaining these in safe and operational condition. This task is seriously hindered by the huge uncertainties that govern the deterioration rate and the lifetime of bridges. In the present work, the time-to-rehabilitation of bridges is probabilistically estimated using data from a large-scale database of the US Federal Highway Administration, which include age and structural condition information for several thousands of bridges. This information reveals the effect of bridge exposure to environmental factors such as deicing salts, traffic loads, earthquakes, humidity and chlorides, among others. The available data are first appropriately processed and adjusted using shifting and scaling procedures. A Weibull distribution function is then fitted to the data to evaluate the probability for a bridge to reach a critical condition and estimate the time-to-rehabilitation. The aforementioned procedure is applied to a real sample of 33,810 concrete bridges of various ages, which are exposed to deicing salts and humidity.
\end{abstract}




\section{INTRODUCTION}

Aging bridges need continuous interventions either in the form of maintenance or major rehabilitation. Reliably estimating the deterioration rate and the lifetime of bridges are essential aspects in determining optimal programs regarding maintenance and/or rehabilitation. This could assist decision makers in both elongating the useful life of bridges and controlling their structural safety in a cost-effective manner. However, the structural performance of bridges in time is governed by huge uncertainties, which need to be quantitatively treated, in order to be able to make rational decisions regarding maintenance and rehabilitation.

An essential step toward the effective handling of such uncertainties is the gathering and exploitation of respective data. Typically, the data collected refer to current bridge conditions and are used in conjunction with Bridge Management Systems (BMS) to decide on the necessity and degree of priority of any potential intervention and to allocate corresponding funds [1]. However, in order to estimate future needs and optimally allocate available budgets, models to predict the future stock condition are essential. Various deterioration models exist and vary from simple linear regression models [2] to much more involved Markov chain models [3,4] and other newer approaches [5-7]. These deterioration models use previous years' structural condition data to trace condition changes and estimate the probability of transition from a condition rating to another.

In the present work, the future structural condition of bridges is probabilistically estimated using real data maintained by the Federal Highway Administration (FHWA) for USA bridges. The National Bridge Inventory (NBI) of FHWA, which is updated annually, contains a considerable amount of information and describes the structural condition of over 500,000 bridges [8]. A single year of the NBI database is used to calibrate a probabilistic model for predicting the structural condition of a bridge over time. Thus, all bridges in the data-stock processed are used, based on their ages, to represent the condition of a single bridge during its lifetime. Hence, the portion of bridges being in a certain age and condition represent the probability of the bridge under study to be in the same condition at that age. This way, curves relating bridge age with cumulative probability for each structural condition can be assembled. Certain time-shifts and scalings are then applied to achieve predictions for bridge ages not covered by available data. By fitting Weibull distribution functions to the original and shifted data and specifying some criteria for deciding bridge rehabilitation, the time left for a bridge until it reaches a structural condition, that induces a need for rehabilitation, can be probabilistically evaluated.

The real NBI sample utilized in this work contains 33,810 concrete bridges of various ages, which are exposed to deicing salts and humidity. Based on this sample, rehabilitation time predictions are calculated. Such predictions can greatly assist in cost-effectively maintaining the desired reliability level of aging bridges.

\section{THE NATIONAL BRIDGE INVENTORY (NBI)}

Inspections are performed on bridges to monitor the infrastructure's stock condition. The FHWA of USA maintains an up-to-date inventory (NBI), which includes over 500,000 bridges in its territory. The NBI contains a considerable amount of information; this includes 116 coded items to describe each bridge. An updated NBI is published annually, as each bridge must be inspected visually biennially. Bridge condition ratings are recorded on a scale 0-9, with 9 representing 'perfect' and 0 'failed' conditions. Inspections are carried out by qualified personnel complying with standard procedures set out in National Bridge Inspection Standards (NBIS) [8].

Condition coded with 5 can be regarded as a threshold for rehabilitation. This corresponds to a 'fair' bridge condition [8]: all primary structural elements are sound but may have minor 
section loss, cracking, spalling or scour. Rehabilitating a bridge in that condition is important, before further deteriorating to a 'poor' (code 4), 'serious' (code 3), 'critical' (code 2), 'imminent failure' (code 1) or 'failed' (code 0) condition.

\section{CASE STUDY: THE UTILIZED BRIDGE STOCK SAMPLE}

The specific case study in this work is related to bridge deterioration with age, e.g. due to corrosion. Deicing salts are known to accelerate deterioration especially in combination with humidity [9]. To locate bridges exposed to chlorides, information from FHWA on the areas of USA using deicing salts have been exploited. The bridges identified are illustrated on the USA map of Fig. 1. To ensure the presence of humidity, the sample was limited to bridges with water passing underneath the structure. Moreover, to limit the structural and material effect on deterioration, only simply supported concrete bridges entered the sample. Finally, cases have been excluded from the selected sample, which correspond to deterioration of a bridge regardless of age. Such cases include earthquakes, accidental actions (e.g. collisions, vandalism), etc. According to the United States Geological Survey (USGS), areas 1 and 2 shown in Fig. 1 represent high seismic hazard regions, which have been excluded from the analysis under study.

Rehabilitated bridges have also been ruled out, as their effect on the sample can be misleading. This is due to the fact that a question arises as to which age to use, based on the year reconstructed or on the year built. In both cases the condition cannot be matched to the rest of the sample, as it is either presented worse or better, respectively.

After the above exclusions the final sample analyzed counted 33,810 bridges.

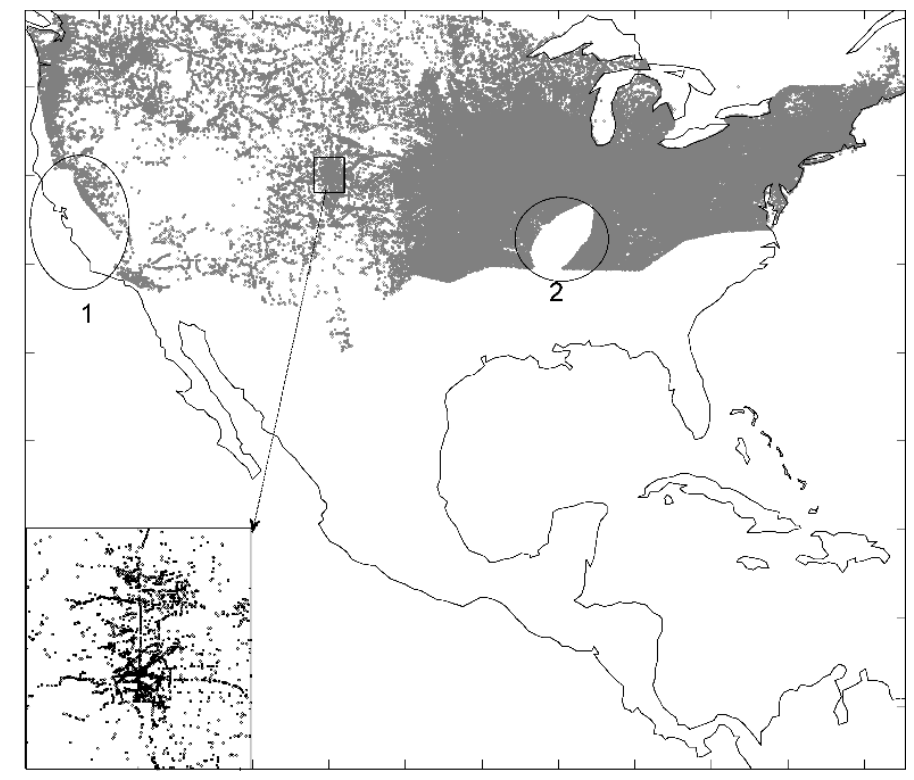

Figure 1: Concrete bridges exposed to deicing salts and humidity. High seismic hazard areas are excluded from the analysis (areas 1 and 2 noted on the figure).

\section{PROCESSING OF BRIDGE STOCK SAMPLE}

In general, the processing of the bridge stock sample described in the previous section is performed as follows. Initially, the probability for a bridge of a certain age to be in a specific structural condition (0-9) is calculated. Then, the residual time to condition 5 is estimated by 
performing time-shifts to future years from condition distributions $>5$. While shifting, the time axis is scaled appropriately. In the next section, Weibull distribution functions are fitted to the original and shifted/scaled data.

The processing of the bridge stock sample is based on the concept of the Cumulative Condition Probability (CCP), which calculates the probability of a bridge of a certain age being equal or below a certain condition. Bridges are categorized in age groups forming sub-stocks of the global bridge stock; the CCP of each sub-stock is independently calculated. CCPs for conditions $\leq 9$ to $\leq 0$ are calculated through:

$$
C C P_{i}(t)=\frac{N_{c, i}(t)}{N_{c, \text { tot }}(t)}=\frac{\sum_{j=0}^{i} N_{j}(t)}{\sum_{j=0}^{9} N_{j}(t)}=\frac{N_{0}(t)+\cdots+N_{i}(t)}{N_{0}(t)+\cdots+N_{9}(t)},
$$

where: $C C P_{i}(t)$ is the CCP for bridge condition $\leq i$ of the sub-stock at age $t ; N_{c, i}(t)$ is the total number of bridges at condition $\leq i$ of the sub-stock at age $t ; N_{c \text {,tot }}(t)$ is the total number of bridges of the sub-stock at age $t$ (at any condition $0-9$ ); $N_{i}(t)$ is the total number of bridges at condition $i$ of the sub-stock at age $t$. Thus, $N_{c, i}$ and $N_{c, \text { tot }}$ are 'cumulative' numbers; this is denoted by the subscript ' $c$ ' used.

The total number of bridges of each bridge sub-stock (i.e. of each year) are shown in Fig. 2, while the corresponding CCPs are illustrated in Fig. 3. Specifically, CCPs for conditions $\leq 9$ to $\leq 3$ are shown in Fig. 3 .

In Fig. 3, it can be noticed that the probabilities (CCPs) calculated through Eq. (1) are not monotonic, but vary within a range, which tends to widen as the bridge sub-stock becomes older. This can be attributed to the smaller samples of constructed bridges available for older years (Fig. 2). It should also be noted that the year of construction may be relevant to the current age of each bridge, but the connection between age and condition has to include the year of inspection. Thus, the actual bridge age that should be considered for calculating CCPs is the year of

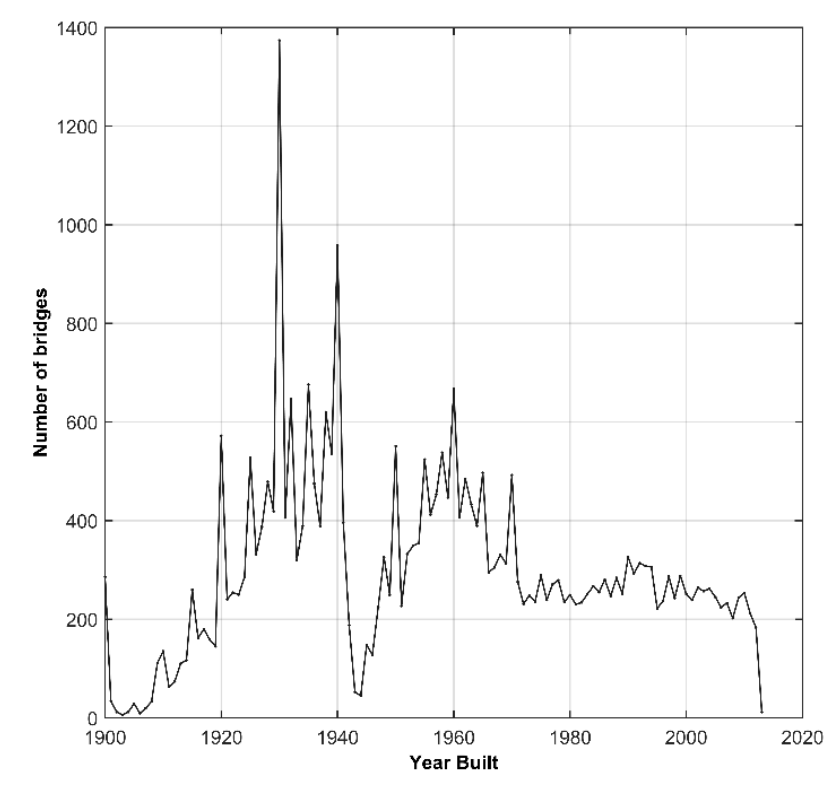

Figure 2: Bridges constructed at each year. 


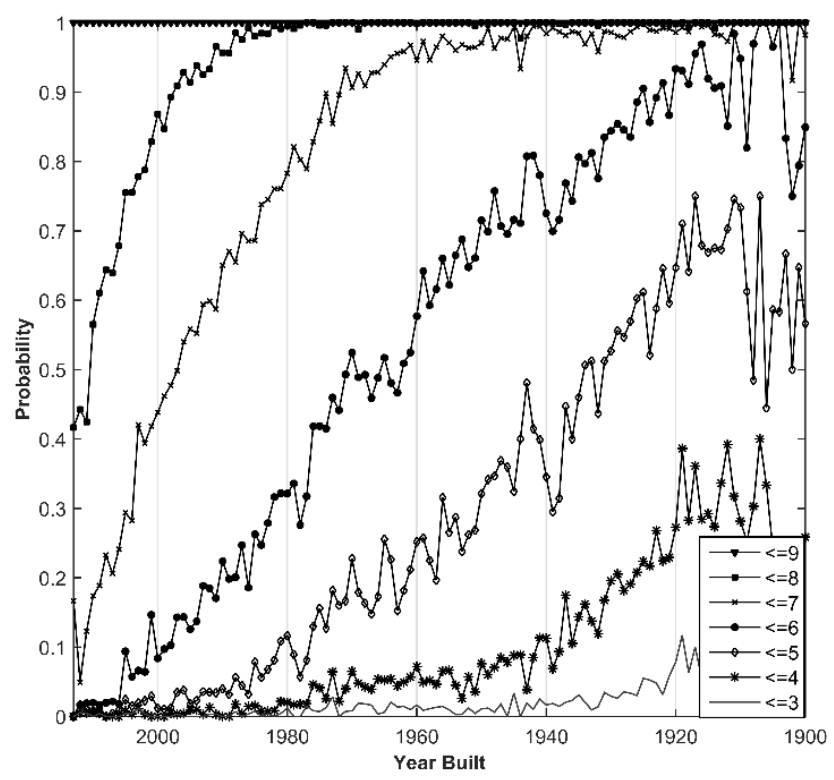

Figure 3: Computed probabilities (CCPs) for each year bridge sample. The 'Year Built' axis is reverse.

inspection subtracted from the year built. By applying this transformation, the graph of Fig. 4 is obtained, in which the variation range of probabilities seems to be lower than in Fig. 3.

To separate the bridge ages with acceptable variation ranges of calculated CCPs, a threshold age $t_{\text {cut }}$ ('cutting age') is determined to define the 'trusted' part of the overall bridge sample (Fig. 4). Probabilities corresponding to bridge sub-stocks older than $t_{c u t}$ are considered to have unacceptably high variation ranges and are therefore 'untrusted'.

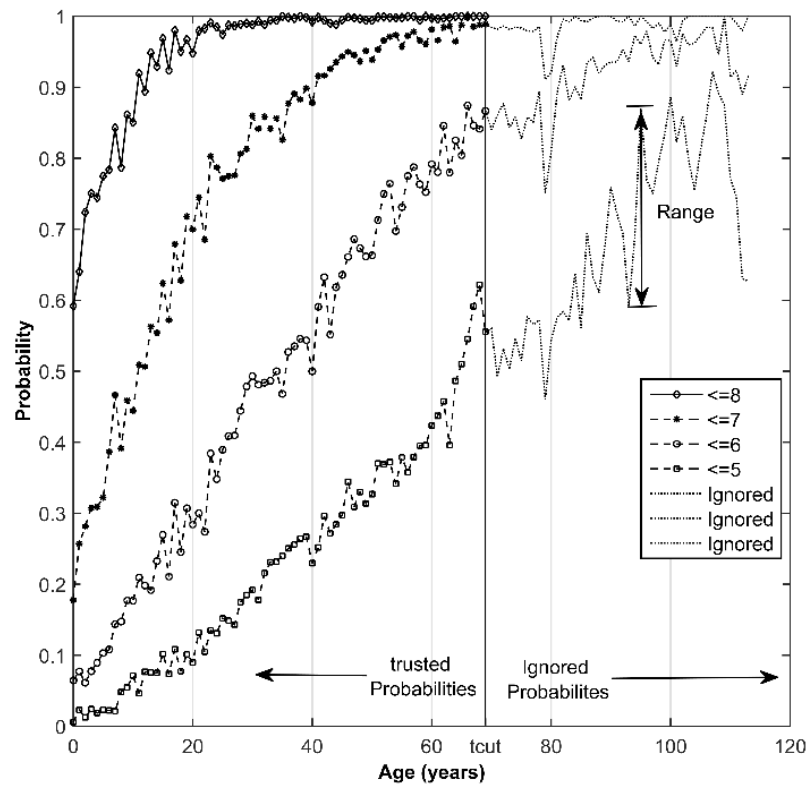

Figure 4: Transformation of probabilities (CCPs) using the year of last inspection; $t_{\text {cut }}$ is used to determine the size of the trusted region. 
Data-shifts are performed to copy data from higher CCPs to lower CCPs, where no trusted data are available. The shifting procedure is illustrated in Fig. 5. Referring, for example, to the CCP-curve for condition $\leq 6$, in order to complete the data beyond the threshold age $t_{\text {cut }}$, data from the CCP-curve for condition $\leq 7$ are used. Hence, point $\mathrm{A}$ is first identified, which is the point with probability $\mathrm{CCP}_{6}\left(t_{c u t}\right)$ at age $t=t_{\text {cut }}$. By drawing a line parallel to the age axis from point A toward the higher $\mathrm{CCP}$-curve, point $\mathrm{B}$ is determined, which is the point of the CCPcurve for condition $\leq 7$ that has the same probability with point A. Point B has probability $C C P_{7}\left(t_{e 7}\right)=C C P_{6}\left(t_{c u t}\right)$ at age $t=t_{e 7}$, thus the age $t_{e 7}$ can be calculated (obviously, $\left.t_{e 7}<t_{c u t}\right)$. Then, the points of the higher $\mathrm{CCP}$-curve from point $\mathrm{B}$ and beyond are horizontally shifted to the lower CCP-curve at point A, i.e. the aforementioned part of the higher CCP-curve is moved toward the lower CCP-curve by $t_{c u t}-t_{e 7}$, as shown by the arrows in Fig. 5. This way, data to have a complete $\mathrm{CCP}$-curve for condition $\leq 6$ are created. The same procedure is performed for every incomplete $\mathrm{CCP}$-curve, in order to be completed beyond the age $t=t_{\text {cut }}$. After performing the required shifts to complete all incomplete curves, all $\mathrm{CCP}$-curves eventually reach the probability-value of one.

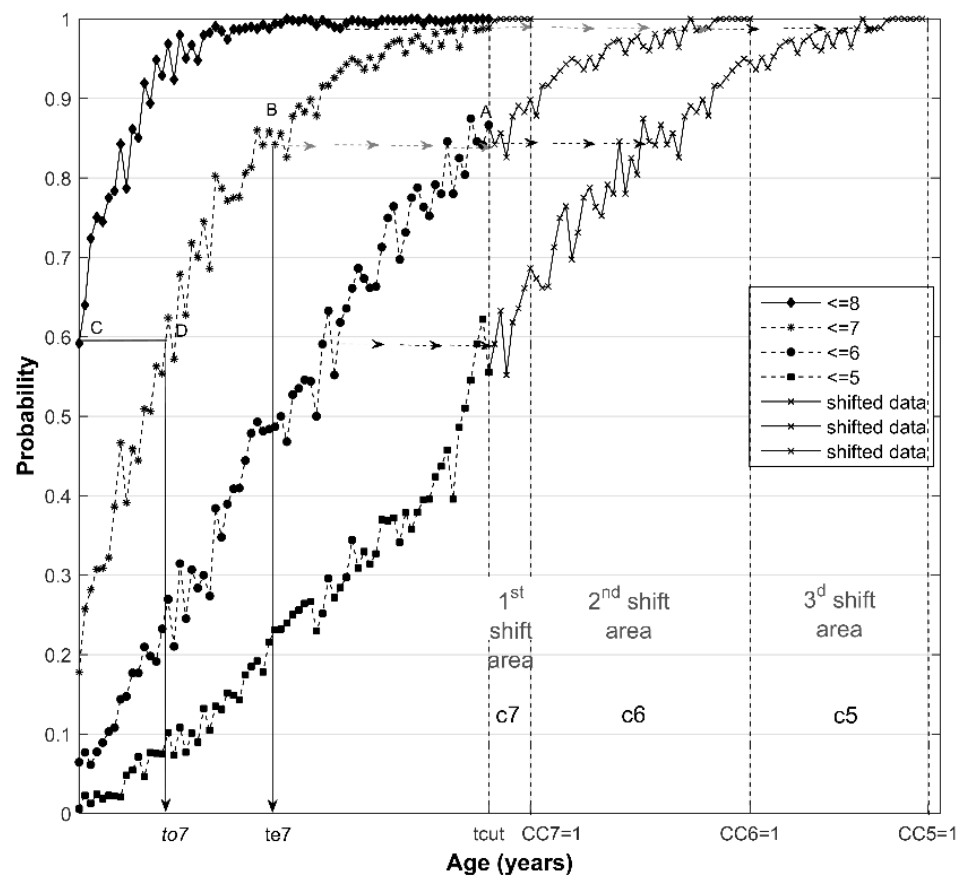

Figure 5: Shifting and scaling procedures.

The shifting procedure described above produces unrealistic high deterioration rates, because data are shifted from higher CCP-curves, which have smaller overall durations (until reaching the probability-value of one). Normally, lower slopes are generally expected for lower $\mathrm{CCP}$-curves than for higher CCP-curves (Figs 3 and 4). Thus, a scaling procedure needs to be also applied, in order to properly adjust the inclination of the part of a CCP-curve that is shifted to complete a lower CCP-curve. This adjustment is performed by horizontally spreading the shifted data with an age scaling procedure, which is realized by introducing the scaling coefficient $c_{i}$ given by: 


$$
c_{i}=\frac{t_{c u t}-t_{\mathrm{o}, i}}{t_{e, i+1}} .
$$

In the above equation, $t_{e, i+1}$ is the age determined during shifting; e.g. when shifting data from the CCP-curve for condition $\leq 7$ to the one for condition $\leq 6, i=6$ and $t_{e, i+1}=t_{e}$. The age $t_{\mathrm{o}, i}$ is evaluated as follows. Referring, for example, to the CCP-curve for condition $\leq 7$ in Fig. 5, point $\mathrm{C}$ is first identified, which is the point of the higher CCP-curve for condition $\leq 8$ at age $t=0$. At point $\mathrm{C}$, the probability is $C C P_{8}(0)$. By drawing a line parallel to the age axis from point $\mathrm{C}$ toward the lower CCP-curve, point $\mathrm{D}$ is determined, which is the point of the CCP-curve for condition $\leq 7$ that has the same probability with point $\mathrm{C}$. Hence, point $\mathrm{D}$ has probability $C C P_{7}\left(t_{07}\right)=C C P_{8}(0)$ at age $t=t_{07}$, thus the age $t_{07}$ can be calculated. Thus, an age $t_{\mathrm{o}, i}$ and a corresponding coefficient $c_{i}$ are calculated for each CCP-curve for condition $\leq i$ having shifted data to be scaled.

Using the calculated scaling coefficients, the spreading of the shifted data is performed for all CCP-curves. Hence, the age range of the shifted data attached to the CCP-curve for condition $\leq i$ is simply multiplied by the coefficient $c_{i}$. Then, the shifted points are spread uniformly within the new, expanded age range resulting in an adjustment of the inclination of the respective CCPcurve. Note that coefficient $c_{i}$ modifies only the shifted age-values and not the corresponding CCP-values.

It should be mentioned that shifts and scalings similar to the ones adopted in the present work are applied to data acquired from accelerated tests performed on materials to evaluate long-term material properties [10].

\section{FITTING OF WEIBULL DISTRIBUTIONS TO THE PROCESSED DATA}

With the purpose of simultaneously obtaining a smooth CCP-curve and also achieving a good description of the respective data, a Weibull distribution [11] can be fitted to the set of CCP-points available for bridge condition $\leq i$. Weibull distributions are extensively used for deterioration modelling and BMS [5]. The standard (two-parameter) Weibull Cumulative Distribution Function (CDF) is given by [11]:

$$
F(t)=1-\exp \left[-\left(\frac{t}{\alpha}\right)^{\beta}\right], t \geq 0,
$$

where $\alpha>0$ is the scale parameter, which has the same unit as $t$, while $\beta>0$ is the unitless shape parameter. Hence, for each CCP-curve, a nonlinear regression is performed to determine the Weibull parameters $\alpha$ and $\beta$ and produce a least-squares fit based on the CDF of Eq. (3).

For the cutting age adopted, Table 1 presents the Weibull parameter values calculated, as well as the coefficient of determination $R^{2}$ attained by applying the fitting procedure to the properly processed CCP-data for bridge conditions $\leq 7, \leq 6$ and $\leq 5$. The very high $R^{2}$-values

\begin{tabular}{llll}
\hline Bridge condition & $\alpha$ (years) & $\beta$ & $R^{2}$ \\
\hline$\leq 7$ & 19.93 & 1.055 & 0.9893 \\
$\leq 6$ & 53.75 & 1.818 & 0.9931 \\
$\leq 5$ & 94.97 & 2.247 & 0.9943 \\
\hline
\end{tabular}

Table 1: Weibull parameter values and goodness-of-fit information. 
reported indicate that a very good fit has been achieved between Eq. (3) and the available data. These successful fits are due to the adequate size of the utilized sample, which exhibits low variation in the available data. In other cases employing lower-quality data, reduced $R^{2}$-values are observed.

\section{PROBABILISTIC ESTIMATION OF THE TIME-TO-REHABILITATION}

Using the Weibull distribution fitted to the set of CCP-points available for bridge condition $\leq 5$, the time-to-rehabilitation can be probabilistically estimated. That is, any time-to-rehabilitation given is accompanied by a probability for the bridge considered to have reached condition $\leq 5$, which is assumed to induce the need for rehabilitation.

Fig. 6 illustrates the three Weibull CDFs obtained and shows a comparison of these curves with the original data. The three CDFs are graphical representations of Eq. (3) using the Weibull parameter values of Table 1 . Hence, the CDF for bridge condition $\leq 5$ provides the sought probabilistic information for the time-to-rehabilitation of a concrete bridge exposed to deicing salts and humidity: by selecting a probability of reaching bridge condition $\leq 5$, the respective timeto-rehabilitation for the bridge is determined. Table 2 gives the time-to-rehabilitation for selected probability-values. Note that, as the term 'time-to-rehabilitation' is used herein, it counts

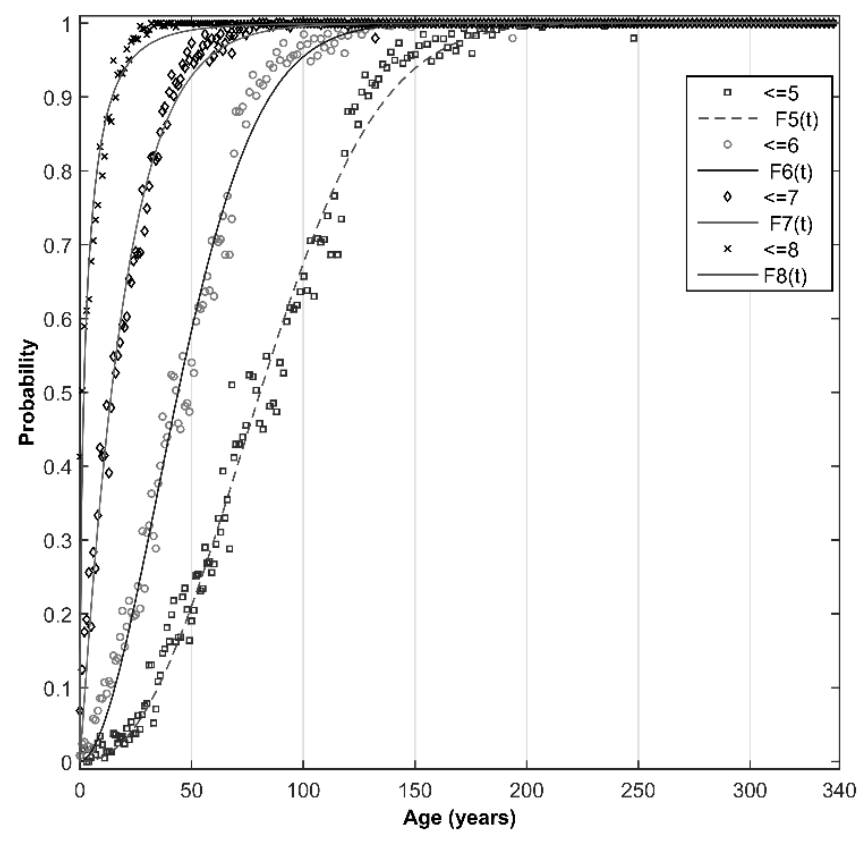

Figure 6: Weibull distributions fitted to the sample data. Original data (before shifting/scaling) are shown for comparison.

\begin{tabular}{lllll}
\hline Probability to reach condition $\leq 5$ & $30 \%$ & $50 \%$ & $70 \%$ & $90 \%$ \\
\hline Time-to-rehabilitation (years) & 60 & 81 & 103 & 138 \\
\hline
\end{tabular}

Table 2: Time-to-rehabilitation for selected probabilities of a bridge to have reached condition $\leqslant 5$. 
from the year a bridge is built, i.e. this is the 'age-to-rehabilitation'. The actual time-to-rehabilitation is easily calculated by subtracting the current age of a bridge from its 'age-to-rehabilitation'. Using such information, a decision maker can make a rational schedule for bridge rehabilitations, which takes into account not only available or anticipated funds, but also risks associated with the uncertain deterioration rate of bridges.

\section{CONCLUSIONS}

In this paper, real condition data from the NBI database of USA' FHWA were utilized for predicting the deterioration of bridges. Specifically, probabilistic results were obtained for concrete bridges exposed to deicing salts and humidity that allow the quantitative estimation of the time-to-rehabilitation. The procedure followed herein can be applied to any type of bridges exposed to various environmental effects, provided that respective data are available. Enriching our knowledge on the deterioration rate of bridge stocks is a key aspect in cost-effectively handling and maintaining such important infrastructures.

\section{REFERENCES}

[1] M.J. Ryall, Bridge management. Butterworth-Heinemann, Oxford, UK, 2001.

[2] M.W. Fitzpatrick, D.A. Law, W.C. Dixon, Deterioration of New York State highway structures. In: Proceedings of 60th Annual Meeting of the Transportation Research Board, Washington D.C., USA, 1981, pp. 1-8.

[3] S. Madanat, R. Mishalani, W.H.W. Ibrahim, Estimation of infrastructure transition probabilities from condition rating data. ASCE Journal of Infrastructure Systems, 1, 120-125, 1995.

[4] S.K. Ng, F. Moses, Prediction of bridge service life using time-dependent reliability analysis. In: Proceedings of 3rd International Conference on Bridge Management, University of Surrey, Guildford, UK, 1996, pp. 26-33.

[5] A.K. Agrawal, A. Kawaguchi, Z. Chen, Deterioration rates of typical bridge elements in New York. ASCE Journal of Bridge Engineering, 15, 419-429, 2010.

[6] Y.H. Huang, Artificial neural network model of bridge deterioration. ASCE Journal of Performance of Constructed Facilities, 24, 597-602, 2010.

[7] M.I. Rafiq, M.K. Chryssanthopoulos, S. Sathananthan, Bridge condition modelling and prediction using dynamic Bayesian belief networks. Structure and Infrastructure Engineering, 11, 38-50, 2015.

[8] Federal Highway Administration (FHWA), Recording and coding guide for the structure inventory and appraisal of the nation's bridges. Report No. FHWA-PD-96-001, Department of Transportation, Washington D.C., USA, 1995.

[9] F. Alogdianakis, I. Balafas, D.C. Charmpis, Environmental effects on bridges - Statistical durability study based on existing inspection data. In: Proceedings of 2 nd International Conference on Innovations on Bridges and Soil-Bridge Interaction (IBSBI 2014), Athens, Greece, 2014, pp. 161-168.

[10] K.G.N.C. Alwis, C.J. Burgoyne, Accelerated creep testing for aramid fibres using the stepped isothermal method. Journal of Materials Science, 43, 4789-4800, 2008. 
[11] G. Yang, Life cycle reliability engineering. John Wiley \& Sons, Hoboken, NJ, USA, 2007. 